\title{
Re-addressing the 2013 consensus guidelines for the diagnosis of insulitis in human type 1 diabetes: is change necessary?
}

\author{
Martha L. Campbell-Thompson ${ }^{1}$ - Mark A. Atkinson ${ }^{1}$ • Alexandra E. Butler ${ }^{2}$. \\ Ben N. Giepmans ${ }^{3}$ - Matthias G. von Herrath ${ }^{4} \cdot$ Heikki Hyöty $^{5}$. Thomas W. Kay ${ }^{6}$. \\ Noel G. Morgan ${ }^{7} \cdot$ Alvin C. Powers $^{8}$ - Alberto Pugliese ${ }^{9} \cdot$ Sarah J. Richardson ${ }^{7}$. \\ Peter A. In't Veld ${ }^{10}$
}

Received: 23 November 2016 / Accepted: 13 December 2016 / Published online: 9 January 2017

(C) Springer-Verlag Berlin Heidelberg 2017

Keywords Human · Inflammation · Insulitis · Islets of Langerhans $\cdot$ Pancreas $\cdot$ Type 1 diabetes $\cdot$ Type 2 diabetes

To the Editor: Lundberg et al [1] propose a 'revised' definition of insulitis in human type 1 diabetes to replace the current consensus guidelines published in 2013 [2]. The authors of this letter, being affiliated with the initial authoritative report, disagree and offer unanimous affirmation and continuing support for the 2013 guidelines.

Martha L. Campbell-Thompson mct@ufl.edu

1 Department of Pathology, Immunology, and Laboratory Medicine, 1395 Center Drive, College of Medicine, University of Florida, Gainesville 32610, FL, USA

2 Larry L. Hillblom Islet Research Center, David Geffen School of Medicine at UCLA, Los Angeles, CA, USA

3 Department of Cell Biology, University of Groningen, Groningen, the Netherlands

4 Department of Developmental Immunology, La Jolla Institute for Allergy and Immunology, La Jolla, CA, USA

5 Department of Virology, University of Tampere and Fimlab Laboratories, Pirkanmaa Hospital District, Tampere, Finland

6 St Vincent's Institute of Medical Research, Fitzroy, VIC, Australia

7 University of Exeter Medical School, Exeter, UK

8 Vanderbilt University Medical Center, Nashville, TN, USA

9 Miller School of Medicine, University of Miami, Miami, FL, USA

10 Department of Pathology, Diabetes Research Center, Vrije Universiteit Brussel, Brussels, Belgium
2013 consensus guidelines for the diagnosis of insulitis in human type 1 diabetes

1. Presence of $\geq 15 \mathrm{CD} 45^{+}$cells immediately adjacent to or within $\geq 3$ islets

2. Predominant lymphocytic infiltration

3. Presence of pseudoatrophic islets (insulinnegative)

To begin, we would note several conflicting and problematic issues with the proposed revised definition of insulitis suggested by Lundberg and colleagues [1]. Specifically, the 2013 guidelines state:

Patients with insulitis are defined by the presence of a predominantly lymphocytic infiltration specifically targeting the islets of Langerhans. The infiltrating cells may be found in the islet periphery (peri-insulitis), often showing a characteristic tight focal aggregation at one pole of the islet that is in direct contact with the peripheral islet cells. The infiltrate may also be diffuse and present throughout the islet parenchyma (intra-insulitis). The lesion mainly affects islets containing insulinpositive cells and is always accompanied by the presence of (pseudo)atrophic islets devoid of beta cells. The fraction of infiltrated islets is generally low $(<10 \%$ of islet profiles). The lesion should be established in a 
minimum of three islets, with a threshold level of $\geq 15$ $\mathrm{CD} 45^{+}$cells/islet before the diagnosis can be made [2]

In their study, Lundberg et al [1] provide a quantitative analysis of leucocyte subsets (CD68, myeloperoxidase [MPO, a marker for neutrophils], CD3 and CD20) in islets from pancreatic organ donors diagnosed with type 1 diabetes, type 2 diabetes and non-diabetic controls, based on pancreas biopsy samples. They conclude that, although the 2013 guidelines led to the identification of insulitis in $31 \%$ of patients with type 1 diabetes in their study, it also identified such lesions in $28 \%$ of their type 2 diabetic patients. Based on this, they propose a revised definition of insulitis. Unfortunately, their conclusions result from a misrepresentation of the 2013 guidelines and would have major implications for those investigating the natural history and pathogenesis of type 1 diabetes. Indeed, the proposed revised guidelines provide no benefit to efforts seeking to uncover the pathogenic events that culminate in type 1 diabetes.

Based on our collective experience examining samples collected from numerous type 1 diabetes patients emanating from a variety of geographical regions, we believe that the proposal by Lundberg et al [1] to use a single criterion for the diagnosis of insulitis (i.e. the presence of $\geq 15 \mathrm{CD}^{+}$cells [rather than $\mathrm{CD} 45^{+}$cells $]$in $\geq 3$ islets) is inappropriate, for several reasons.

First and foremost, we believe the proposal to use the term 'insulitis' in the context of type 2 diabetes is not only misleading, but also quite incorrect, as there are no data to support the proposition that insulitis exists and plays a role in the pathogenesis of type 2 diabetes. Indeed, taken to its fullest, we view such a notion as being counter-productive as collectively we are attempting to define the pathogenesis of type 1 diabetes for the purpose of identifying a cure.

Beyond this, we note the following: the 2013 insulitis guidelines state explicitly that the presence of pseudoatrophic islets (i.e. islets devoid of beta cells) is required before making a diagnosis of insulitis. The presence of such islets is a strong indication of an autoimmune process with beta cell specific destruction. Therefore, inclusion of this parameter is a principal safeguard to avoid the inaccurate diagnosis of insulitis when high levels of infiltrating leucocytes are present diffusely in the pancreatic parenchyma, as in chronic pancreatitis. The proposed revised definition does not explicitly take the presence of pseudoatrophic islets into account and therefore weakens markedly the 2013 guidelines. In fact, if the combined criteria defined in the 2013 guidelines (i.e. $\geq 15 \mathrm{CD} 45^{+}$cells in $\geq 3$ islets, a predominantly lymphocytic infiltrate and the presence of pseudoatrophic islets) are applied to the data in the Lundberg paper [1], they are perfectly able to distinguish the true insulitis in four patients with recent onset type 1 diabetes from the generalised $\mathrm{CD} 45^{+} / \mathrm{CD} 68^{+}$ cell infiltration seen in the group of type 2 diabetic patients, especially as the latter do not display pseudoatrophic islets.

Lundberg et al propose the use of $\mathrm{CD} 3$ as a marker for infiltrating $\mathrm{T}$ lymphocytes, rather than CD45 (a more common leucocyte marker recognising all T cell subsets, B lymphocytes, macrophages and nucleated leucocytes) as used in the 2013 consensus guidelines [1]. However, the use of CD3 would preclude the detection of $\mathrm{CD} 20^{+} \mathrm{B}$ lymphocytes and macrophages, cell types shown to be present in infiltrated islets in both children and adults with type 1 diabetes [3-5]. The 2013 consensus guidelines, therefore, although recognising that the majority of infiltrating cells are of a lymphocytic nature, specifically propose to use the CD45 marker in immunotyping to ensure that the contribution of other key immune cell types to the insulitic infiltrate would not be missed, as this could lead to underestimation of the prevalence and severity of insulitis.

Furthermore, and in contrast to long-established practice, Lundberg et al propose to use the term insulitis in the context of type 2 diabetes as well as type 1 , with insulitis being diagnosed in patients in whom $\geq 15 \mathrm{CD} 45^{+}$cells are found in $\geq 3$ islets. We believe that this proposal is misleading as there is currently insufficient data indicating that both types of diabetes have a common immune pathogenesis. It is important to note that the infiltrates described by Lundberg et al in the context of type 2 diabetes appear to be diffuse, not islet specific, and not predominantly of a lymphocytic nature. The major proportion of infiltrating cells in such islets appear to consist of macrophages. The infiltrates shown in Fig. 1a-d of the Lundberg study [1] would not be considered to be insulitis by the consensus definition, but rather they represent an extension of inflammation from the surrounding parenchyma. Chronic pancreatitis in patients with type 2 diabetes is well known and the fibrotic areas reported in the Lundberg paper (Fig. 1c, d) clearly show such regions [6].

The revised definition proposes to use the term insulitis in the context of both type 1 and type 2 diabetes and is likely to increase confusion within the field. We believe that clarity is of paramount importance when defining insulitis, since further advances in our understanding of the aetiopathogenesis of type 1 diabetes will rely on robust definitions that are applied rigorously. In stating this, the expert group also affirms that any revision of the guidelines be based on objective and improved criteria that provide mechanistic insight into the disease process. However, in our view, the proposals by Lundberg et al do not provide such an improvement. Therefore, after careful consideration, we stand united in support of the 2013 consensus guidelines and reaffirm their validity.

Funding Work in the authors' laboratories was supported by the JDRF (25-2013-268, 17-2012-3 and 25-2012-516 [MC-T, MAA, AP, 2-SRA2015-68-Q-R [ACP]) and NIH (DP3 DK101120-01 [MC-T, MAA], P01 AI42288 [MAA, MC-T], UC4 DK 10415501 [MAA, MC-T], DK106755, DK104211, DK108120 [ACP], European Union's Seventh Framework Programme PEVNET [FP7/2007-2013] under grant agreement number 261441. JDRF Career Development Award (5-CDA-2014221-A-N [SJR]), Diabetes UK (15/0005156 [NGM, SJR]). 
Duality of interest The authors declare that there is no duality of interest associated with this manuscript.

Contribution statement All authors were responsible for drafting the article and revising it critically for important intellectual content. All authors approved the version to be published.

\section{References}

1. Lundberg M, Seiron P, Ingvast S, Korsgren O, Skog O (2017) Insulitis in human diabetes: a histological evaluation of donor pancreases. Diabetologia 60:346-353
2. Campbell-Thompson ML, Atkinson MA, Butler AE et al (2013) The diagnosis of insulitis in human type 1 diabetes. Diabetologia 56:2541-2543

3. Campbell-Thompson M, Fu A, Kaddis JS et al (2016) Insulitis and $\beta$-cell mass in the natural history of type 1 diabetes. Diabetes 65:719-731

4. Leete P, Willcox A, Krogvold L et al (2016) Differential insulitic profiles determine the extent of $\beta$-cell destruction and the age at onset of type 1 diabetes. Diabetes 65:1362-1369

5. Coppieters KT, Dotta F, Amirian N et al (2012) Demonstration of islet-autoreactive CD8 T cells in insulitic lesions from recent onset and long-term type 1 diabetes patients. J Exp Med 209:51-60

6. Ewald N, Kaufmann C, Raspe A, Kloer HU, Bretzel RG, Hardt PD (2012) Prevalence of diabetes mellitus secondary to pancreatic diseases (type 3c). Diabetes Metab Res Rev 28:338-342 